\title{
A amamentação e o risco de transmissão de COVID-19
}

\author{
Breastfeeding and the risk of transmission of COVID-19
}

\author{
Lactancia materna y el riesgo de transmisión de COVID-19
}

\begin{abstract}
Bruna de Oliveira Godoi ${ }^{1 *}$, Camylla Cristina de Melo Alvino ${ }^{1}$, Evelyn Caldas dos Santos ${ }^{1}$, Karen Iulianne Machado da Silva ${ }^{1}$, Jackson Gois Teixeira ${ }^{1}$, Brenda Christina Vieira ${ }^{1}$, Silvana Dias de Macedo França ${ }^{1}$, Gabriela Ataides de Oliveira ${ }^{1}$, Flávia Miquetichuc Nogueira Nascente ${ }^{1}$, Albênica Paulino dos Santos Bontempo'.
\end{abstract}

\section{RESUMO}

Objetivo: Observar o risco de transmissão da COVID-19 durante a amamentação e os principais cuidados necessários em sua execução. Métodos: Revisão sistemática, realizada em julho de 2020, na Biblioteca Virtual em Saúde, com os descritores "Lactante", "Recém-Nascido" e "COVID-19", disponíveis na íntegra. Assunto principal: infecção por coronavírus, publicados em 2020. Resultados: Foram localizados 172 artigos; após remoção dos duplicados, indisponíveis, uso dos filtros e critérios de elegibilidade, restaram 9 para análise. Observou-se que o leite materno é o melhor alimento para o neonato, sendo importante também para o desenvolvimento do seu sistema imunológico. Quanto à COVID-19, as evidências foram insuficientes para afirmar a transmissão do vírus, embora não tenha sido encontrado nas amostras de leite analisadas. Há controvérsias nos textos quanto aos riscos e benefícios da amamentação no contexto pandêmico. No entanto, muitos autores recomendam o alojamento conjunto e a prática da amamentação. Considerações Finais: Não há evidências robustas da presença do vírus no leite materno ou do risco de transmissão durante a amamentação. Recomenda-se que as puérperas infectadas sejam orientadas quanto aos cuidados para prevenir que gotículas com vírus cheguem ao neonato por vias aéreas. As informações encontradas na literatura são limitadas, é necessário que mais estudos sejam realizados.

Palavras-chave: Lactante, Recém-nascido, COVID-19.

\begin{abstract}
Objective: To observe the risk of transmission of COVID-19 during breastfeeding and the main cares needed in its execution. Methods: Systematic review, carried out in July 2020, at the Virtual Health Library with the descriptors "Lactant", "Newborn" and "COVID-19", fully available. Main theme: Coronavirus infection, published in 2020. Results: 172 articles were found; After removing duplicates, unavailable ones, use of filters and eligibility criteria, 9 has left for analysis. It was observed that breast milk is the best for newborn feeding, being also important for his immune system development. Regarding COVID-19, not enough evidences were found to confirm virus transmissions, although it was not found in the analyzed milk sample. There are controversial in the texts related to risks and benefits of breastfeeding in a pandemic context. However, many authors recommend joint accommodation and breast feeding practice. Final Considerations: There is no robust evidence of the presence of the virus on the breast milk or of the risk of transmission during breastfeeding. It is recommended that puerperal women are oriented about the care to prevent droplets with the virus reach the newborn from the airways. The information found in the literature is limited and further studies are needed.
\end{abstract}

Keywords: Lactant, Newborn, COVID-19.

${ }^{1}$ Centro Universitário do Distrito Federal (UDF), Brasília - DF. *E-mail: bruna.oliveiragodoii@gmail.com 


\section{RESUMEN}

Objetivo: Observar el riesgo de transmisión de COVID-19 durante la lactancia y los principales cuidados necesarios en su ejecución. Métodos: Revisión sistemática, realizada en julio de 2020, en la Biblioteca Virtual en Salud con los descriptores "Lactante", "Recién nacido" y "COVID-19", disponible en su totalidad, tema principal: Infección por coronavirus, publicado en 2020. Resultados: Se encontraron 172 artículos; Después de eliminar dos duplicados no disponibles, utilizo dos filtros y criterios de elegibilidad para restaurar 9 para su análisis. Se señaló que la alimentación materna o neonatal también es importante para el desarrollo del sistema inmunológico. Sin embargo, con respecto al COVID-19, no se encontró evidencia suficiente en la literatura analizada para confirmar la transmisión del virus. Existen textos controvertidos sobre la edad de los riesgos y beneficios de la lactancia materna en un contexto de pandemia. Sin embargo, muchos autores recomiendan la acomodación conjunta y la práctica de enfermería. Consideraciones Finales: No existe evidencia sólida de la presencia del virus en el lado materno o del riesgo de transmisión durante la lactancia. Se recomienda que las madres infectadas reciban atención durante cuántos años para evitar que las gotitas de virus pasen por las vías respiratorias hasta el recién nacido. La información encontrada en la literatura es limitada y se necesitan más estudios.

Palabras clave: Lactante, Recién nacido, COVID-19.

\section{INTRODUÇÃO}

Os coronavírus humanos MERS-CoV, SARS-CoV e SARS-CoV-2 têm sido a causa de infecções graves, provocando, respectivamente, a Síndrome Respiratória do Oriente Médio (MERS), a Síndrome Respiratória Aguda Grave (SARS) e doença infecciosa por coronavírus 2019 (COVID-19) (CHEN Y, et al., 2020). Este último é responsável por um surto iniciado na cidade de Wuhan, na China, em dezembro de 2019. A Organização Mundial de Saúde (OMS) declarou emergência de saúde pública de interesse internacional em janeiro de 2020 (WHO, 2020) e, posteriormente, uma pandemia em março do mesmo ano (WHO, 2020), uma vez que o número de casos confirmados de COVID-19 já ultrapassava 2 milhões em todo o mundo (WHO, 2020).

Estudos revelam que a transmissão do SARS-CoV-2 ocorre principalmente de pessoas para pessoas através de gotículas respiratórias (CHAN JFW, et al., 2020; GHINAI I, et al., 2020). Também existem evidências emergentes de que o vírus pode ser detectado em fluidos biológicos, incluindo fluidos de lavagem broncoalveolar (WANG W, et al., 2020), sangue (WANG W, et al., 2020; YOUNG BE, et al., 2020) e saliva (TO KKW, et al., 2020), mas ainda existem lacunas substanciais no conhecimento sobre a presença de SARSCoV-2 no leite materno. Neste contexto, mulheres grávidas com COVID-19 têm uma difícil escolha a fazer após o parto: se devem ou não amamentar seus filhos (VOGEL G, 2020).

O leite materno é o alimento mais completo para o bebê, pois fortalece a imunidade da criança e favorece a prevenção de doenças respiratórias e infecciosas, além de ser uma técnica de baixo custo financeiro e simples (RODRIGUES NA e GOMES ACG, 2014). No entanto, existe a possibilidade de também ser um canal para a transmissão de doenças infecciosas, fato este que pode gerar grande medo e ansiedade nas lactantes e até mesmo conduzir a interrupção do aleitamento materno. Existem evidências na literatura que demonstraram a transmissão vertical de várias estirpes bacterianas, vírus e parasitas através da amamentação (FRANCO C, et al., 2018).

Considerando o atual momento pandêmico, não há evidências robustas de transmissão vertical do coronavírus entre a mãe infectada e seu neonato. Não se sabe se o vírus pode ser transmitido para o feto durante a gestação ou durante o trabalho de parto, muito embora alguns poucos estudos tenham afirmado que não encontraram o vírus no líquido amniótico (PERONI DG e FANOS V, 2020). No entanto, é sabido que uma mãe infectada pode transmitir a COVID-19 através de gotículas respiratórias durante o contato íntimo (GIULIANI C, et al., 2020).

Diante do exposto, e considerando a grande relevância deste tema, o presente estudo tem por objetivo observar através de uma revisão sobre o risco de transmissão da COVID-19 durante a amamentação e as principais recomendações para execução correta do aleitamento materno durante a pandemia por meio de uma revisão sistemática. 


\section{MÉTODOS}

Revisão sistemática, realizada em julho de 2020, nas seguintes bases de dados: Literatura Latinoamericana e do Caribe em Ciências da Saúde (LILACS), Scientific Electronic Library Online (SCIELO), Medical Literature Analysis and Retrieval System Online (MEDLINE), WEB OF SCIENCE, BANCO DE TESES DA CAPES, Cumulative Index to Nursing and Allied Health Literature (CINAHL) e SCOPUS, acessadas por meio da Biblioteca Virtual em Saúde - BVS. Os descritores extraídos do Medical Subject Headdings (MeSH) utilizados em português foram "Lactante", "Recém-Nascido" e "COVID-19". Com o intuito de refinar a pesquisa, optou-se pelo uso dos seguintes filtros: "texto disponível na íntegra", "assunto principal - infecção por Coronavírus", publicados em 2020. Realizaram a busca de forma independente três pesquisadores, que seguiram as orientações do "Relatório Preferidos para Revisões Sistemáticas e Meta-Análises" (PRISMA).

A seleção seguiu os critérios de elegibilidade, priorizando artigos associados à relação da amamentação com a contaminação pela contaminação do novo coronavírus causador da doença COVID-19 considerando o binômio mãe e filho. Foram excluídos estudos sobre medicamentos, vacinas, exames, artigos de opinião e cartas editoriais.

Todo o processo teve três etapas: no primeiro momento, houve a leitura dos títulos, em seguida foi realizada a leitura dos resumos dos documentos previamente selecionados, e, finalmente, a leitura dos artigos na íntegra para compilação dos achados. Todas as etapas foram realizadas simultaneamente pelos pesquisadores que, somente após o término da busca independente, compararam seus resultados e discutiram as divergências, de modo que apenas os documentos aprovados pelos três pesquisadores fizessem parte do presente estudo. Por fim, foram extraídos os resultados e as conclusões mais relevantes de cada artigo.

\section{RESULTADOS}

Foram localizados 172 artigos; logo em seguida, foram removidos os documentos duplicados e indisponíveis na íntegra. Utilizou-se então filtros e foram aplicados critérios de elegibilidade, de modo que restaram apenas 9 artigos para etapa de análise crítica. Os detalhes da busca estão esquematizados no fluxograma da Figura 1.

Figura 1 - Fluxograma descrevendo a estratégia de busca e seleção de estudos dos itens Preferenciais de Relatórios para Revisões Sistemáticas e Meta-Análises (PRISMA): Diagrama de fluxo 2019.

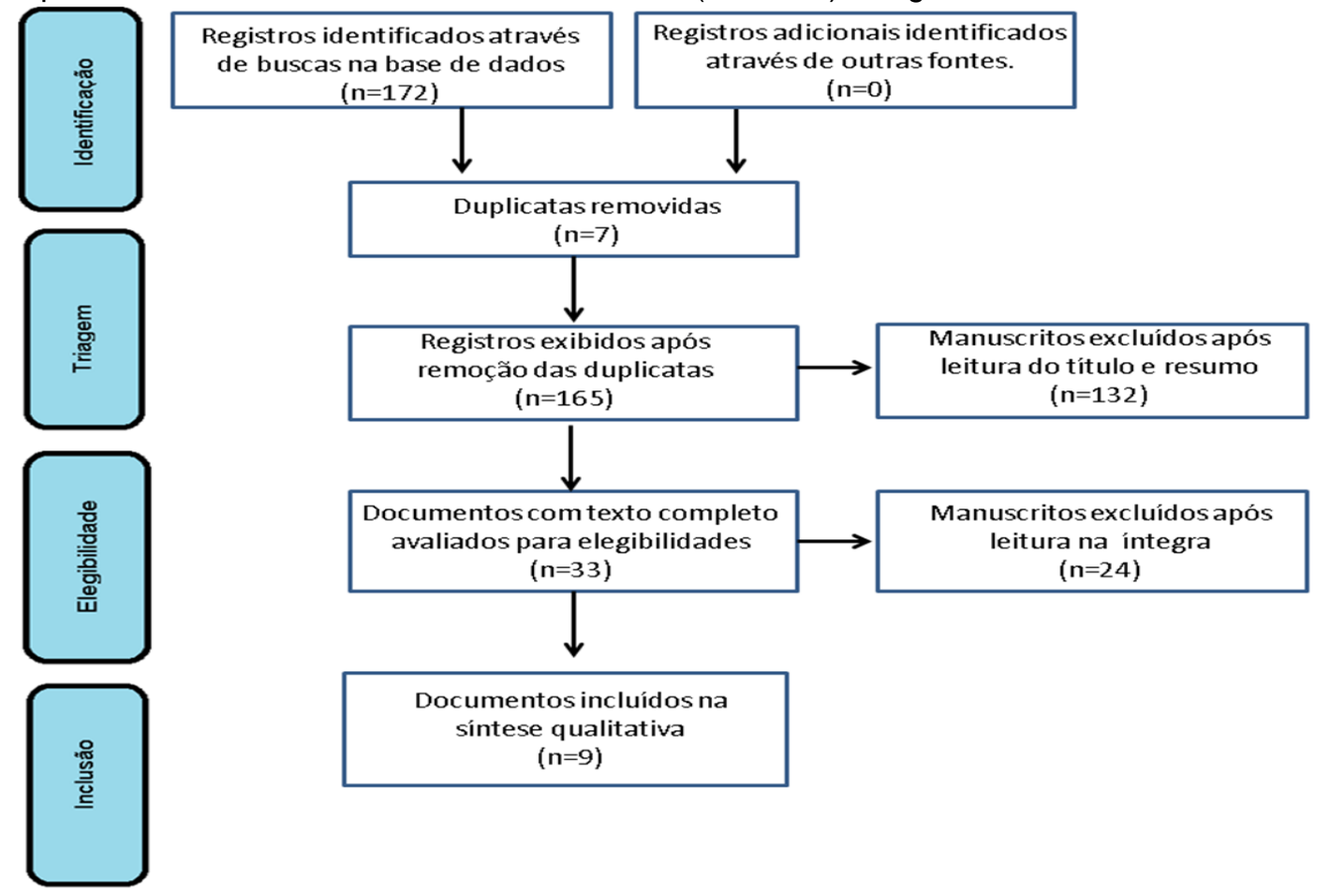

Fonte: Godoi BO, et al., 2020. 
O Quadro 1 é um compilado das principais informações retiradas dos nove artigos que estavam de acordo com os critérios de elegibilidade e foram selecionados para o presente estudo referentes a relação da amamentação e o risco de transmissão da COVID-19 entre a mãe infectada e seu neonato. Neste quadro observa-se a síntese dos principais achados de cada um dos artigos.

Quadro 1 - Síntese dos principais achados sobre a relação da amamentação com a contaminação pelo COVID-19. Brasília-Brasil, 2020.

\begin{tabular}{|c|c|c|}
\hline $\mathbf{N}$ & Autores (Ano) & Principais achados \\
\hline 1 & YANG P, et al., 2020. & $\begin{array}{l}\text { Estudo retrospectivo. As características clínicas, os dados laboratoriais e os } \\
\text { resultados de } 7 \text { neonatos de gestantes COVID-19 positivo (submetidas a parto } \\
\text { cesáreo) mostraram que não houve transmissão vertical; no entanto, os autores } \\
\text { sugerem a separação imediata como prevenção. }\end{array}$ \\
\hline 2 & $\begin{array}{l}\text { RASMUSSEN SA, et } \\
\text { al., } 2020 .\end{array}$ & $\begin{array}{l}\text { Revisão sistemática. Não houve evidência de transmissão vertical, no entanto as } \\
\text { gestantes observadas foram contaminadas no } 3^{\circ} \text { trimestre e submetidas a parto } \\
\text { cesáreo. Os autores afirmam que a amostra foi pequena para determinar as } \\
\text { recomendações da amamentação baseadas em evidências. }\end{array}$ \\
\hline 3 & $\begin{array}{c}\text { LANG GJ e ZHAO H, } \\
2020 .\end{array}$ & $\begin{array}{l}\text { Relato de caso de gestante COVID-19 positivo. Imediatamente após o parto } \\
\text { cesáreo, o neonato foi submetido ao teste de RT-PCR, sendo negativo. As } \\
\text { análises repetidas do leite materno foram negativas. Os autores sugerem que a } \\
\text { prática da amamentação ocorra após o período de isolamento. }\end{array}$ \\
\hline 4 & PENG Z, et al., 2020. & $\begin{array}{l}\text { Relato de caso de gestante COVID-19 positivo, contaminada no } 3^{\circ} \text { trimestre, parto } \\
\text { cesáreo. Os autores declararam que é necessário ter cautela ao concluir que a } \\
\text { transmissão vertical é improvável. No entanto, os pesquisadores afirmam que o } \\
\text { neonato foi negativo para o vírus e não foi encontrado vírus no líquido amniótico, } \\
\text { secreções vaginais, sangue do cordão umbilical, placenta, soro, cotonete anal e } \\
\text { leite materno. }\end{array}$ \\
\hline 5 & $\begin{array}{c}\text { DAVANZO R, et al., } \\
2020 .\end{array}$ & $\begin{array}{l}\text { Recomendações da SIN e UENPS. Mãe COVID-19 positivo, assintomática e } \\
\text { paucissintomática pode amamentar o neonato em regime de alojamento conjunto, } \\
\text { sob estritas medidas de segurança. Caso a mãe esteja muito doente para cuidar } \\
\text { do neonato, ele será tratado separadamente e alimentado com leite materno } \\
\text { fresco, sem necessidade de pasteurizacão. }\end{array}$ \\
\hline 6 & STUEBE A, 2020. & $\begin{array}{l}\text { Recomendações da OMS e CDC. A separação da mãe e neonato não é } \\
\text { recomendada, uma vez que pode não prevenir a infecção, além de estressar as } \\
\text { mães, perturbar a fisiologia do neonato e interferir no fornecimento de leite } \\
\text { materno, prejudicando a proteção imunológica inata e específica. Não receber a } \\
\text { amamentação amplia o risco de hospitalização do neonato por pneumonia, e o } \\
\text { isolamento separado dobra a carga sobre o sistema de saúde. }\end{array}$ \\
\hline 7 & $\begin{array}{l}\text { PROCIANOY RS, et } \\
\text { al., } 2020 .\end{array}$ & $\begin{array}{l}\text { Recomendações da SBP, MS e PRN. Mãe e neonato (a termo) estáveis devem } \\
\text { permanecer em alojamento conjunto isolado e receber orientação sobre medidas } \\
\text { de segurança e uso de EPIs. Se ambos adoecerem, devem permanecer } \\
\text { separados e considerar a intenção da mãe de amamentar por meio da extração } \\
\text { do leite. }\end{array}$ \\
\hline 8 & $\begin{array}{c}\text { RONDELLI G, et al., } \\
2020 .\end{array}$ & $\begin{array}{l}\text { Recomendações da ABENFO e SOBEP. O aleitamento materno deve ser mantido } \\
\text { em casos de suspeita ou confirmação da infecção } \\
\text { materna, desde que a mulher apresente o desejo de amamentar e que esteja } \\
\text { em condições clínicas adequadas para fazê-lo; a mãe deve receber orientação } \\
\text { sobre medidas de segurança e uso de EPIs. }\end{array}$ \\
\hline 9 & $\begin{array}{l}\text { MIMOUNI F, et al., } \\
2020 .\end{array}$ & $\begin{array}{l}\text { Recomendações da CDC. Mães em isolamento devem ser encorajadas a } \\
\text { ordenhar o leite para estabelecer e manter o suprimento, utilizando bomba de } \\
\text { sucção, após higiene das mãos. O leite materno ordenhado pode ser oferecido ao } \\
\text { recém-nascido por um cuidador saudável. Caso a mãe e o neonato permaneçam } \\
\text { juntos e a mãe deseje amamentar no peito, ela deverá ser orientada quanto às } \\
\text { medidas de segurança e uso de EPIs. }\end{array}$ \\
\hline
\end{tabular}

Legenda: ${ }^{*} \mathrm{RT}-\mathrm{PCR}=$ Reverse transcription polymerase chain reaction. ${ }^{*} \mathrm{SIN}=$ Sociedade Italiana de Neonatologia; *UENPS = Sociedades Neonatal e Perinatal da União Europeia; *OMS = Organização Mundial da Saúde; ${ }^{\star} \mathrm{CDC}=$ Centros de Controle e Prevenção de Doenças; ${ }^{*} \mathrm{SBP}=$ Sociedade Brasileira de Pediatria; ${ }^{*} \mathrm{MS}=$ Ministério da Saúde; ${ }^{*} \mathrm{PRN}=$ Programa de Reanimação Neonatal; ${ }^{*} E P I s=$ Equipamento de Proteção Individual. ABENFO = Associação Brasileira de Enfermeiros Obstetras e Obstetrizes; ${ }^{\star}$ SOBEP = Sociedade Brasileira de Enfermeiros e Pediatras.

Fonte: Godoi BO, et al., 2020. 


\section{DISCUSSÃO}

O presente estudo possibilitou apresentar um mapeamento sobre a produção de conhecimento disponível acerca do risco de transmissão da COVID-19 durante o processo de amamentação, além de observar as principais recomendações de grandes instituições renomadas sobre a prática segura do aleitamento materno uma vez que à medida em que a pandemia do coronavírus está progredindo, as mulheres gestantes infectadas experimentam grande medo e incertezas quanto aos cuidados necessários para com seus filhos após o parto.

Embora as evidências científicas apontem que a maioria das infecções causados pelo coronavírus sejam leves, há, no entanto a necessidade de dar maior atenção na assistência a saúde das gestantes levando em consideração fatos históricos como os que aconteceram em outras epidemias severas semelhantemente a que está acontecendo agora, nas últimas duas décadas, a exemplo do ocorrido no Oriente Médio com a síndrome respiratória MERS-COV em que cerca de um terço das mulheres grávidas infectadas não resistiram e vieram a óbito (HAMED MA, 2020).

Deste modo é de suma importância o atendimento às populações vulneráveis como as gestantes em cenários assustadores como esse, que engloba uma doença com transmissibilidade tão elevada. Uma vez que as mulheres gestantes são afetadas desproporcionalmente por doenças respiratórias, as quais estão relacionadas a altas taxas de mortalidade materna assim como apresentam também elevada taxa de aborto espontâneo, teratogenicidade ou restrição de crescimento intra-uterino (HAMED MA, 2020; CHEN H, et al., 2020).

No estudo de Liang H e Acharya G (2020), os autores afirmam que a gravidez é como um estado de imunossupressão parcial que torna as mulheres mais vulneráveis a infecções virais e o Ministério da Saúde (2020) diz que este fato está relacionado com as mudanças fisiológicas que ocorrem durante a fase da gestação e podem com facilidade desenvolver uma rápida progressão em casos de doenças respiratórias. Ainda assim, o estudo de Herraiz I, et al. (2020) demostrou que as mulheres grávidas são grupo de risco relativamente baixo para COVID-19, uma vez que são jovens e saudáveis, que geralmente são assintomáticas, no entanto, ainda existem incertezas sobre os efeitos que o vírus pode causar na gestante, feto e neonato.

Considerando os sintomas da Covid-19 apresentados pelas mulheres durante o período gestacional, muitos estudos demonstram que os mais relatados foram febre, calafrios, tosse sem expectoração, mialgia, dor de garganta, dor leve na parte superior do abdômen, no tórax, nas costas, mal-estar/fadiga e em alguns casos dispepsia. Além destes sintomas também foram relatados casos de sofrimento fetal e linfopenia. Até o presente momento sabe-se que a infecção e inflamação materna que ocorre em virtude da infecção viral, pode afetar o feto no primeiro trimestre da gestação. Após o princípio da gestação não foram observadas alterações relevantes relacionadas a mãe e o bebê, embora não seja descartada a possibilidade de ocorrer transmissão vertical e em alguns casos nascimentos prematuros (CHEN H, et al., 2020).

Neste contexto, a equipe de assistência a lactante precisa orienta-la devidamente para que esta não tome decisões sem uma base de evidências e corra o risco de influenciar negativamente as interações mãe-filho e até mesmo gerar comprometimentos futuros severos, uma vez que a amamentação é essencial para a saúde de bebês e crianças pequenas (WANG L, et al., 2020). Tendo em vista que há na literatura fortes evidências de que o colostro é o 'alimento perfeito' para os recém-nascidos, e grandes instituições como a OMS recomendam que a amamentação seja iniciada logo após o parto, preferencialmente dentro do período de uma hora (ERBAYDAR NP e ERBAYDAR T, 2020).

A amamentação é extremamente importante para a saúde tanto das mães quanto de seus bebês. $O$ conselho atual é que a amamentação exclusiva seja estimulada até que os bebês alcancem os seis meses de idade. Há estudos que afirmam que bebês alimentados com leite artificial correm maior risco de infecções, asma e síndrome da morte súbita infantil, assim como mães que não amamentam também estão sujeitas a um maior risco de desenvolver câncer e diabetes (FAIR FJ, et al., 2019). 
O leite humano é considerado a fonte de nutrição ideal para bebês nascidos a termo durante os primeiros seis meses de vida, fornecendo nutrientes necessários para seu crescimento e desenvolvimento. Ele também fornece fatores antimicrobianos e imunomoduladores, que servirão na defesa contra infecções. Portanto, a prática da amamentação possibilita a exposição do bebê ao sistema imunológico da mãe durante a fase crucial dos primeiros meses de vida, quando seu sistema imunológico se encontra ainda em desenvolvimento. No entanto, a composição imunomoduladora de leite materno humano não foi bem caracterizada e demonstra uma grande variabilidade entre as mães (JARVINEN KM, et al., 2019).

Todas estas informações apresentadas até aqui são argumentos plausíveis da importância de realizar uma maior reflexão acerca do potencial comprometimento da COVID-19 e das recomendações necessárias para que mães e filhos não sofram qualquer impedimento desnecessário em seu direito de usufruir dos benefícios da amamentação com a devida segurança.

Logo, diante da pergunta norteadora do presente estudo foi possível observar um fato que não fazia parte da investigação inicial, mas que de modo algum poderia ser ignorado por estar intimamente relacionado à amamentação. Observou-se que gestantes infectadas foram submetidas a parto cesáreo como forma de prevenção da contaminação do neonato e da equipe de assistência (YANG P, et al., 2020; RASMUSSEN SA, et al., 2020; LANG GJ e ZHAO H, 2020; PENG Z, et al., 2020).

O tipo de parto está entre os muitos fatores que afetam as práticas de amamentação. Além disso, há um crescimento constante do número de estudos publicados que relatam as consequências negativas da cesariana sobre o bem-estar e o comportamento das novas mães e a fisiologia da lactação durante o período pós-parto. A cesariana é considerada uma cirurgia abdominal de grande porte, e procedimentos de rotina no pós-cirúrgico da puérpera podem atrasar a amamentação na primeira hora após o parto. Poucas horas após a cesariana, espera-se que as novas mães comecem a cuidar de seus recém-nascidos enquanto lidam simultaneamente com os problemas associados ao período pós-operatório, incluindo a dor pós-cirúrgica, o que pode afetar negativamente na amamentação inicial (ERBAYDAR NP e ERBAYDAR T, 2020).

Sobre a via de parto, Yang $P$, et al. (2020) relataram em seu estudo a realização de partos cesáreos foi realmente escolhida como uma forma de prevenção da contaminação do feto e da equipe de assistência. No entanto, nos estudos realizados por Baergen RN e Heller DS (2020) e Jie P e Xinghui L (2020), os pesquisadores relataram casos de parto espontâneo em que as mães COVID-19 positivo puderam permanecer com seus bebês e com os pais na sala de parto, sem complicações. Esses autores ainda orientam que, na escolha da via de parto, deve-se considerar as condições obstétricas da parturiente e as condições de segurança da equipe.

Segundo Chen $\mathrm{H}$, et al. (2020), a via de parto não necessariamente precisa ser cesárea, mas é recomendada em casos de hipóxia materna, choque séptico e/ou sofrimento fetal. O Ministério da Saúde não recomenda a ocorrência de partos na água, para segurança da equipe e do neonato, uma vez que o vírus também é eliminado nas fezes (BRASIL, 2020).

Considerando a transmissão vertical do vírus, observou-se que até o momento não há evidências robustas de que o vírus seja transmitido através do leite materno (WHO, 2020; CDC, 2020). No entanto, em ambos os estudos realizados por Yang P, et al. (2020) e Rasmussen SA, et al. (2020), os autores afirmaram que as amostras estudadas foram pequenas demais para determinar as recomendações acerca da amamentação baseadas em evidências.

Em contrapartida, o estudo realizado por Zimmermann P e Curtis N (2020) sugere a possibilidade de transmissão vertical da COVID-19 e, portanto, acredita que o contato próximo durante a amamentação ou 0 contato da mãe com o recém-nascido podem causar transmissão por gotículas. Entretanto, Rondelli G, et al. (2020) acredita que, em caso de suspensão da oferta do leite materno, os recém-nascidos serão privados dos benefícios desta prática e estarão sujeitos a desencadear outros problemas de saúde a curto e longo prazo. Ao considerar as evidências disponíveis, os benefícios do aleitamento materno parecem superar os riscos. 
O relato de caso de Peng Z, et al. (2020), realizado na China, mostrou que durante a internação de uma mãe COVID-19 positivo e seu neonato, foi realizada uma série de testes nucleicos de SARS-CoV-2 no leite materno, e os resultados foram todos negativos. Semelhantemente, no estudo de Lang GJ e Zhao H (2020), outro relato de caso, os autores afirmaram que o teste de análise de presença do vírus no leite materno também foi negativo. Vale ressaltar que, embora as amostras dos casos clínicos analisados sejam pequenas, ainda assim merecem atenção por trazerem as evidências disponíveis neste momento para o enfrentamento dessa doença no que diz respeito à amamentação.

Com o intuito de prevenir a infecção cruzada e reduzir as chances de contaminação dos neonatos de mães infectadas, o estudo de Yang P, et al. (2020) orienta que, após o nascimento, os bebês sejam separados de suas mães temporariamente. Já nos estudos de Davanzo R, et al. (2020) e Procianoy RS, et al. (2020), os autores afirmam que é possível que mãe e neonato permaneçam em regime de alojamento conjunto e devese considerar a intenção da mãe de amamentar, seja no seio ou por meio da extração do leite.

Segundo Stuebe A (2020), o aleitamento materno deve ser mantido mesmo quando há suspeita ou confirmação da infecção materna, desde que a mulher apresente o desejo de amamentar e que esteja em condições clínicas adequadas para fazê-lo (PROCIANOY RS, et al., 2020). Mimouni F, et al. (2020) descreveram as recomendações do Centros de Controle e Prevenção de Doença (CDC) e dizem que tanto as mães podem ordenhar o leite para que um cuidador saudável possa alimentar seu filho quanto podem elas mesmas amamentarem. Caso permaneçam juntos e a mãe deseje amamentar no peito, ela deverá ser orientada quanto às medidas de segurança e uso de EPIs, tais como aventais, luvas, máscaras cirúrgicas e proteção ocular, para evitar que gotículas com carga viral cheguem ao bebê, e deve ser suspendido o contato cutâneo (CARVALHO WB, et al., 2020).

Nos estudos de Stuebe A (2020), Procianoy RS, et al. (2020), Rondelli G, et al. (2020) e Mimouni F, et al. (2020), os autores apresentam as principais recomendações de grandes instituições como a OMS, SIN, UENPS, CDC, SBP, MS, PRN, ABENFO e SOBEP acerca da amamentação em caso de puérperas com suspeita ou confirmação de infecção. Assim, recomenda-se o alojamento conjunto com medidas de segurança e a prática da amamentação, mas deve-se sempre considerar o desejo da mãe e orientá-la sobre o uso de EPIs.

Embora a maioria dos autores recomendem a prática de aleitamento materno, os efeitos do vírus no início da gravidez são completamente desconhecidos, os números para determinar as recomendações da amamentação baseadas em evidências são muito pequenos e é necessário que os obstetras se mantenham atualizados sobre a transmissão em sua área de atuação e nas orientações nacionais (RASMUSSEN SA, et al., 2020).

O leite materno é sem dúvidas o melhor alimento para o recém-nascido, além disso contém uma variedade de componentes biologicamente ativos que aparentemente agem como moduladores do desenvolvimento do sistema imunológico neonatal (LE DOARE K, et al., 2018). Quanto à COVID-19, as evidências disponíveis são insuficientes para afirmar a transmissão do vírus e há controvérsias encontradas nos textos quanto aos riscos e benefícios da amamentação no contexto pandêmico.

Durante a elaboração deste documento, surgiu a necessidade de abordar outro assunto de extrema importância relacionado ao aleitamento materno e à COVID-19. O fato é que as puérperas COVID-19 positivo em tratamento fazem uso de medicamentos para o controle da infecção, logo, por este estudo tratar das principais recomendações para a amamentação, faz-se necessário discorrer sobre o tema. Assim, é preciso relatar que muitos estudos estão em desenvolvimento com várias drogas para saber qual teria eficácia científica, mas ainda não há uma terapêutica efetiva contra a COVID-19.

Enquanto isso, a cloroquina, que é um agente antimalárico usado na prevenção e tratamento da malária e em algumas síndromes imunológicas, como lúpus eritematoso sistêmico e artrite reumatóide, tem sido prescrita por alguns médicos para lactantes, de modo que há estudos que afirmam que foram encontradas quantidades muito pequenas de cloroquina excretadas no leite materno e que não foram observados efeitos contrários no lactente após o uso por suas nutrizes. Sabe-se que este medicamento possui um alto volume de distribuição, o que sugere níveis muito baixos no leite materno (CHAVES RG, et al., 2020). 
Outro medicamento utilizado no tratamento da COVID-19 é a azitromicina, um antibiótico do grupo dos macrolídeos que também apresenta baixos níveis no leite materno; seu uso é seguro em lactentes. Semelhantemente, a ivermectina, um fármaco indicado na intervenção de doenças parasitárias, também tem sido usada contra o coronavírus. Os estudos sobre seu uso por lactantes são limitados, mas indicam que a ivermectina é pouco excretada no leite materno e a concentração consumida pelo lactente é tão pequena que não é esperada a ocorrência de efeitos adversos (CHAVES RG, et al., 2020).

Diante do exposto, infere-se que os medicamentos atualmente indicados para o tratamento da COVID-19 não apresentam contraindicação de uso pela lactante, o que possibilita associar o tratamento com 0 aleitamento em mães que apresentarem condições clínicas para amamentar ou extrair o leite materno (CHAVES RG, et al., 2020).

\section{CONSIDERAÇÕES FINAIS}

Até o momento, não foram encontradas evidências robustas que comprovem a presença do vírus SARSCoV-2 no leite materno e o risco de transmissão durante a amamentação. No entanto, recomenda-se que as puérperas infectadas sejam orientadas quantos aos cuidados necessários para prevenir que gotículas com carga viral cheguem ao neonato; para isso, devem fazer uso de equipamento de proteção individual. As informações encontradas na literatura são limitadas. São necessários mais estudos, com grandes amostras, para que esses resultados sejam confirmados.

\section{REFERÊNCIAS}

1- BAERGEN RN, HELLER DS. Placental pathology in Covid-19 positive mothers: preliminary findings. Pediatric and Developmental Pathology, 2020; 23(3): 177-180.

2- BRASIL. Ministério da Saúde. Recomendações para o trabalho de parto, parto e puerpério durante a pandemia da covid-19: Nota técnica n ${ }^{\circ}$ 9/2020 - COSMU/CGCIVI/DAPES/SAPS/MS. Disponível em: https://portaldeboaspraticas.iff.fiocruz.br/wp-content/uploads/2020/04/SEI_MS-0014382931-NotaTecnica_9.4.2020_parto.pdf. Acesso em: 18 nov. 2020.

3- CARVALHO WB, et al. Expert recommendations for the care of newborns of mothers with COVID-19. Clinics, 2020; 75: E1932.

4- CENTER FOR DISEASE CONTROL AND PREVENTION. 2020. In: CDC United States: Pregnancy, Breastfeeding, and Caring for Newborns. Disponível em: https://www.cdc.gov/coronavirus/2019-ncov/need-extraprecautions/pregnancybreastfeeding.html?CDC_AA_refVal=https\%3A\%2F\%2Fwww.cdc.gov\%2Fcoronavirus\%2F2019ncov\%2Fprepare\%2Fpregnancy-breastfeeding.html. Acesso em: 27 set. 2020.

5- CHAN JFW, et al. A familial cluster of pneumonia associated with the 2019 novel coronavirus indicating person-toperson transmission: a study of a family cluster. Lancet, 2020; 395(10223): 514-23.

6- CHAVES RG, et al. Aleitamento materno e terapêutica para a doença coronavírus 2019 (COVID-19). Resid Pediatr, $2020 ; 10(2): 1-6$.

7- $\mathrm{CHEN} \mathrm{H}$, et al. Clinical characteristics and intrauterine vertical transmission potential of COVID-19 infection in nine pregnant women: a retrospective review of medical records. The Lancet, 2020; 395(10226): 809-815.

8- CHEN Y, et al. Emerging coronaviruses: Genome structure, replication, and pathogenesis. J Med Virol, 2020; 92(4): 418-423.

9- DAVANZO R, et al. Breastfeeding and coronavirus disease-2019: Ad interim indications of the Italian Society of Neonatology endorsed by the Union of European Neonatal \& Perinatal Societies. Maternal \& Child Nutrition, 2020; 16(3): e13010.

10- ERBAYDAR NP, ERBAYDAR T. Relationship between caesarean section and breastfeeding: evidence from the 2013 Turkey demographic and health survey. BMC Pregnancy and Childbirth, 2020; 20(1): 55.

11- FAIR FJ, et al. Intervenções para apoiar o início e a continuação da amamentação entre mulheres com sobrepeso ou obesas. Cochrane Database of Systematic Reviews, 2019; 9(9): CD012099.

12- FRANCO C, et al. Transmissão de Infeções pelo Aleitamento Materno. Acta Pediatr Port, 2018; 49: $243-52$.

13- GHINAI I, et al. First known person-to-person transmission of severe acute respiratory syndrome coronavirus 2 (SARS-CoV-2) in the USA. The Lancet, 2020; 395(10230):1137-1144.

14- GIULIANI C, et al. Breastfeeding during the COVID-19 pandemic: suggestions on behalf of Woman Study Group of AMD. Diabetes Research and Clinical Practice, 2020; 165(108239).

15- HAMED MA. COVID-19 An overview on: reality and expectation. Nature Public Health Emergency Collection, 2020; 44(1): 86. 
16- HERRAIZ I, et al. Universal screenig for SARS-Cov-2 before labor admission during Covid-19 pandemic in Madrid. Journal of Perinatal Medicine, 2020; 48(9): 981-984.

17- JARVINEN KM, et al. Immunomodulatory effects of breast milk on food allergy. Annals of Allergy, Asthma \& Immunology, 2019; 123(2): 133-143.

18- JIE P, XINGHUI L. Classification management recommendations for the full prevention and control of perinatal period under the outbreak of new coronavirus pneumonia. Chinese Journal of Obstetrics and Gynecology, 2020; 55(00): E005-E005.

19- LANG GJ, ZHAO H. Can SARS-CoV-2-infected women breastfeed after viral clearance?. J Zhejiang Univ Sci B, 2020; 21(5): 405-407.

20- LE DOARE K, et al. Mother's milk: a purposeful contribution to the development of the infant microbiota and immunity. Frontiers in immunology, 2018; 9:361.

21- LIANG H, ACHARYA G. Novel Coronavirus disease (COVI-19) in pregnacy: What clinical recommendations to follow?. Obstetrics \& Gynecology, 2020; 99(4): 439-442.

22- MIMOUNI F, et al. Perinatal aspects on the covid-19 pandemic: a practical resource for perinatal-neonatal specialists. Journal of Perinatology, 2020; 40(5): 820-826.

23- PENG Z, et al. Unlikely SARS-CoV-2 vertical transmission from mother to child: A case report. Journal of infection and Public Health, 2020; 13(5): 818-220.

24- PERONI DG, FANOS V. Lactoferrin is an important factor when breastfeeding and COVID-19 are considered. Acta Paediatrica, 2020; 109(10): 2139-2140.

25- PROCIANOY RS, et al. Neonatal COVID-19: little evidence and the need for more information. Jornal de Pediatria, 2020; 96(3): 269-272.

26- RASMUSSEN SA, et al. Coronavirus Disease 2019 (COVID-19) and pregnancy: what obstetricians need to know. Am J Obstet Gynecol, 2020; 222(5): 415-426.

27- RODRIGUES NA, GOMES ACG. Aleitamento Materno: Fatores Determinantes do Desmame Precoce. Enfermagem Revista, 2014; 17(1): 30-48.

28- RONDELLI G, et al. Assistência às gestantes e recém-nascidos no contexto da infecção Covid-19: Uma revisão sistemática. Desafios - Revista Interdisciplinar da Universidade Federal do Tocantins, 2020; 7(Especial-3): 48-74.

29- STUEBE A. Should infants be separated from mothers with COVID-19? First, do no harm. Breastfeeding Medicine, 2020; 15(5): 351-352.

30- TO KKW, et al. Consistent detection of 2019 novel coronavirus in saliva. Clinical Infectious Diseases, 2020; 71(15): 841-843.

31- VOGEL G. 2020. In: SCIENCE: New coronavirus leaves pregnant women with wrenching choices-but little data to guide them. Disponível em: https://www.sciencemag.org/news/2020/03/new-coronavirus-leaves-pregnant-womenwrenching-choices-little-data-guide-them. Acesso em: 27 set. 2020.

32- WANG L, et al. Chinese expert consensus on the perinatal and neonatal management for the prevention and control of the 2019 novel coronavirus infection. Annals of translational medicine, 2020; 8(3): 47.

33- WANG W, et al. Detection of SARS-CoV-2 in different types of clinical specimens. Jama, 2020; 323(18): $1843-1844$.

34- WORLD HEALTH ORGANIZATION. 2020. In: WHO United States: Coronavirus disease (COVID-19) Pandemic: Public advice and Country technical guidance. Disponível em: https://www.who.int/emergencies/diseases/novelcoronavirus-2019. Acesso em: 28 set. 2020.

35- WORLD HEALTH ORGANIZATION. 2020. In: WHO United States: Director-General's opening remarks at the media briefing on COVID-19. Disponível em:https://www.who.int/dg/speeches/detail/who-director-general-s-openingremarks-at-the-media-briefing-on-covid-19---11-march-2020. Acesso em: 28 set. 2020.

36- WORLD HEALTH ORGANIZATION. 2020. In: WHO United States: New FAQs address healthcare workers questions on breastfeeding and COVID-19. Disponível em: https://www.who.int/news/item/28-04-2020-new-faqs-addresshealthcare-workers-questions-on-breastfeeding-and-covid-19. Acesso em: 27 set. 2020.

37- WORLD HEALTH ORGANIZATION. 2020. In: WHO United States: Statement on the second meeting of the International Health Regulations (2005) Emergency Committee regarding the outbreak of novel coronavirus (2019nCoV). Disponível em: https://www.who.int/news/item/30-01-2020-statement-on-the-second-meeting-of-theinternational-health-regulations-(2005)-emergency-committee-regarding-the-outbreak-of-novel-coronavirus-(2019ncov). Acesso em: 21 jul. 2020.

38- YANG P, et al. Clinical characteristics and risk assessment of newborns born to mothers with COVID-19. Journal of Clinical Virology, 2020; 127(104356).

39- YOUNG BE, et al. Epidemiologic features and clinical course of patients infected with SARS-CoV-2 in Singapore. Jama, 2020; 323(15):1488-1494.

40- ZIMMERMANN P, CURTIS N. COVID-19 in children, pregnancy and neonates: a review of epidemiologic and clinical features. The Pediatric infectious disease journal, 2020; 39(6): 469-477. 